\title{
Herméneutique contre dialectique : la rhétorique argumentative dans La Thébaïde, Britannicus, Mithridate
}

Delphine Reguig

\section{CpenEdition}

Journals

Édition électronique

URL : http://journals.openedition.org/rhetorique/96

DOI : $10.4000 /$ rhetorique.96

ISSN : 2270-6909

Éditeur

UGA Éditions/Université Grenoble Alpes

Édition imprimée

ISBN : 978-2-84310-255-4

Référence électronique

Delphine Reguig, « Herméneutique contre dialectique : la rhétorique argumentative dans La Thébaïde, Britannicus, Mithridate », Exercices de rhétorique [En ligne], 1 | 2013, mis en ligne le 06 novembre 2013, consulté le 12 septembre 2020. URL : http://journals.openedition.org/rhetorique/96 ; DOI : https:// doi.org/10.4000/rhetorique.96

Ce document a été généré automatiquement le 12 septembre 2020.

\section{c) (7)(2) (2)}

Les contenus de la revue Exercices de rhétorique sont mis à disposition selon les termes de la Licence Creative Commons Attribution - Pas d'Utilisation Commerciale - Partage dans les Mêmes Conditions 4.0 International. 


\title{
Herméneutique contre dialectique : la rhétorique argumentative dans La Thébaïde, Britannicus, Mithridate
}

\author{
Delphine Reguig
}

1 Dans la Pratique du théâtre, l'abbé d'Aubignac écrit de la scène que «c'est où règne le Démon de l'inquiétude, du trouble et du désordre ${ }^{1} »$ et ce critique loue les délibérations élevées par le dramaturge au rang des discours pathétiques qui, loin de n'être que de longues suites de raisons rationnellement ordonnées, "portent le caractère Théâtral », c'est-à-dire, "sont impétueux et par les raisonnements et les figures ${ }^{2}$ ». Tout poète tragique relève le défi formulé par d'Aubignac affirmant d'une part que la tragédie, imitation d'action par le discours, est, par nature, contraire aux « discours » qui ne sont pas de ses « appartenances » et définissant d'autre part le discours tragique comme une action même. C'est dans ce cadre qu'il faut examiner la manière dont l'argumentation se trouve, chez Racine ${ }^{3}$, enchâssée dans la structure dramatique de l'action de telle sorte qu'elle participe de son dynamisme. La nécessité de l'argumentation s'impose au genre tragique en tant qu'il met en scène le conflit. J. Lecompte, dans sa thèse, rappelle ainsi que la contradiction des volontés des différents acteurs est une caractéristique fondamentale de la tragédie dans la définition proposée par le Père Rapin dans ses Réflexions sur la Poétique ${ }^{4}$. Le conflit garantit « l'agitation » qui, pour Rapin comme pour d'Aubignac, maintient l'attachement du spectateur à la représentation. Dans ce cadre, l'argumentation doit aussi s'analyser comme le lieu d'exploration privilégié des rapports entre rhétorique externe et rhétorique interne ${ }^{5}$ elle met certes en jeu la persuasion des spectateurs mais surtout la mise en scène de la persuasion des personnages les uns par les autres, c'est-à-dire la transformation du discours en objet de spectacle pour ceux que d'Aubignac appelle les "regardants ${ }^{6}$ ». On a beaucoup souligné l'omniprésence chez Racine de la rhétorique telle qu'Aristote la définit au chapitre II de la Rhétorique, la manière d'évaluer pour chaque question «ce qui peut être propre à persuader ", l'art de considérer «ce qui est propre à persuader ", la recherche des preuves, «des ressources pour procurer des raisons ${ }^{7}$ » de croire. La Thébaïde s'ouvre ainsi sur l'ambition persuasive de Jocaste : 


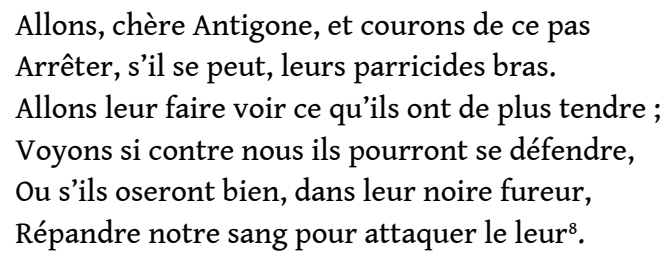

2 Burrhus et Narcisse se disputent la persuasion de Néron, et Mithridate, entrant sur scène, entend se soustraire à tout effort persuasif :

Princes, quelques raisons que vous me puissiez dire, Votre devoir ici n'a point dû vous conduire,

Ni vous faire quitter, en de si grands besoins,

Vous, le Pont, vous, Colchos, confiés à vos soins. [Britannicus, II, 2, v. 423-429]

3 Or, la perspective ouverte par les spécialistes de la rhétorique racinienne (P. France, G. Declercq, M. Hawcroft) consiste à souligner à la fois la compétence technique de personnages représentés en orateurs et leur incompétence rhétorique fondamentale, manifeste dans leur impuissance à rendre leur discours persuasif par incapacité notamment à s'adapter à leur auditeur. Cette impossible adaptation est constatée par les spectateurs qui se trouvent en mesure d'apprécier et de goûter, comme un plaisir esthétique, ce que G. Declercq nomme " le spectacle d'impuissance rhétorique » donné par la tragédie, celui d'« une rhétorique sans finalité qui perd de vue son interlocuteur pour s'exercer sur l'orateur lui-même ${ }^{9}$ ».

4 L'intensité pathétique de ce spectacle se trouve majorée, dans nos trois pièces en particulier, par l'instauration d'une dimension seconde de l'argumentation. Car si le spectateur, par le procédé de l'ironie tragique, est mis en position d'assister à l'échec persuasif des personnages ${ }^{10}$, il voit cette analyse anticipée et comme sans cesse tentée par les personnages eux-mêmes. Le dispositif rhétorique intrascénique est lui-même organisé de telle sorte que l'argumentation, dans ses procédés comme dans sa finalité, se trouve faire l'objet de l'évaluation des personnages entre eux. Cette évaluation intrascénique de l'argumentation des personnages par les personnages se produit en fonction de la logique, de la pertinence, de l'efficacité de cette argumentation. Cette figuration est également essentielle pour la représentation de l'argumentation, c'est-àdire sa transformation en objet scénique. C'est là un élément supplémentaire de tension dramatique et de production du pathétique car l'examen des raisons des personnages les uns par les autres contribue au blocage de l'action et donc à l'impasse que l'argumentation avait l'ambition, précisément, de surmonter.

\section{Le procédé et sa finalité}

Le métadiscours argumentatif, dans les pièces que nous allons étudier, ne s'absorbe pas dans le seul effort de réfutation ; l'argumentation ne suscite pas seulement la contreargumentation, elle devient le support d'une argumentation au second degré : plutôt que de répondre aux arguments présentés, pour instaurer une dialectique, les personnages répondent par un commentaire sur l'argumentation présentée. Dans un dédoublement lui aussi rhétorique entre la parole scénique et l'intention réelle qui lui présiderait, l'argumentation est présentée comme un ensemble de "procédés ", de moyens artificiels qui ne servent qu'une ambition pragmatique déliée de tout souci de vérité. Ce qui se trouve à l'œuvre, c'est le déploiement récurrent de l'argument de la dissociation entre le procédé utilisé par l'orateur et sa sincérité. Les personnages 
raciniens se reprochent ainsi de méconnaître le précepte de Quintilien selon lequel: "L'essentiel [...] est que prévale auprès de nous ce que nous voulons voir prévaloir chez le juge, et que nous soyons touchés nous-mêmes avant d'essayer de toucher les autres ${ }^{11}$ ». Cette distance entre l'orateur et son discours est une faute argumentative qui nourrit l'évaluation de l'argumentation des personnages par leurs auditeurs. Cette évaluation se trouve utilisée comme une archi-preuve intrinsèque au discours, retournée contre l'argumentation elle-même, désarmant et "traversant ${ }^{12}$ " les « efforts ${ }^{13}$ » de persuasion déployés par les caractères.

6 Cette diversion s'enracine dans la conscience, exprimée sur la scène, des limites pragmatiques de la persuasion. Réduit à néant par l'usage récurrent du mot « rien » dans $L a$ Thébaïde ${ }^{14}$, l'effort persuasif se trouve dramatisé par sa dénonciation même ${ }^{15}$. En conclusion de la scène IV, 3 de La Thébaïde, Jocaste en fait l'amer constat :

Allez donc, j'y consens, allez perdre la vie ;

À ce cruel combat tous deux je vous convie;

Puisque tous mes efforts ne sauraient vous changer,

Que tardez-vous? [Thébaïde, v. 1179-1181]

Ce constat soutient lui-même une surenchère pathétique où l'argumentation se trouve littéralement exténuée pour confiner à la provocation du scandale logique [Thébaïde ,v. 1183-1184] : «Surpassez, s'il se peut, les crimes de vos pères;/ Montrez, en vous tuant, comme vous êtes frères. »

8 Pour Narcisse, Néron figure cet abandon de la tentative argumentative lorsqu'il la remplace par un portrait de lui-même en orateur défait; face à Agrippine, l'empereur n'a plus de voix, son discours s'atrophie et cette «nudité » de l'orateur démuni s'exprime dans une éthopée paradoxale qui passe par les procédés du portrait pour précisément exprimer l'inefficacité du discours :

Éloigné de ses yeux, j'ordonne, je menace,

J'écoute vos conseils, j'ose les approuver;

Je m'excite contre elle, et tâche à la braver :

Mais (je t'expose ici mon âme toute nue)

Sitôt que mon malheur me ramène à sa vue,

Soit que je n'ose encor démentir le pouvoir

De ces yeux où j'ai lu si longtemps mon devoir ;

Soit qu'à tant de bienfaits ma mémoire fidèle

Lui soumette en secret tout ce que je tiens d'elle,

Mais enfin mes efforts ne me servent de rien:

Mon génie étonné tremble devant le sien. [Britannicus, II, 2, v. 495-505]

Néron se représente en orateur muet, donne à voir le mutisme qui l'affecte lorsqu'il est soumis au regard d'Agrippine. Le pouvoir de ce regard entrave toute expression de l'individu dans ce portrait pathétique d'un empereur dépendant qui ne peut qu'atteindre le spectateur, ce «regardant» tout puissant assistant au progrès de l'action en dépit des argumentations déployées contre elle.

10 Vaine, l'argumentation est aussi dénoncée comme un faux-semblant, une stratégie hypocrite dissimulant l'effet des passions, l'exercice d'une sophistique utilitariste au service de l'intérêt qui prend le nom de "chimère ", " déguisements ", "traverses ", " mystère ${ }^{16}$ ». Face à elle, on se livre à des reformulations du discours d'autrui : corrections, déductions, prolongements et conversions argumentatifs, inversion des rôles argumentatifs (notamment dans le couple accusé/victime ${ }^{17}$ ) sont les instruments de cette paraphrase ${ }^{18}$. Cette dernière sert une évaluation en acte qui questionne la 
vraisemblance et donc l'efficacité de l'argumentation en se substituant à une réponse elle-même argumentée sur la pertinence des arguments et des preuves mobilisées. L'exposition de Mithridate repose sur ces procédés de progression par transgression du pacte rhétorique : on refuse de prendre l'argumentation pour ce qu'elle veut être et on la considère comme une diversion ou une manœuvre dilatoire. À l'acte I, scène 3 , Pharnace presse ainsi Monime de ne plus attendre son père et de s'unir à lui ; Monime lui oppose un discours où elle ressaisit l'unité de son caractère ( "Je crois que je vous suis connue », v. 247), rappelle son identité et récapitule son histoire. Elle construit le dernier épisode de cette narration, le projet de mariage avec Mithridate et le meurtre de son père par les Romains en représailles, de telle manière qu'il fasse apparaître un conflit d'intérêts entre une éventuelle union avec Pharnace et la fidélité à la mémoire paternelle [v. 266-274] : «Enfin, je n'ai qu'un cœur. Tout ce que je puis faire, / C'est de garder la foi que je dois à mon père, / De ne point dans son sang aller tremper mes mains / En épousant en vous l'allié des Romains. » Elle invoque ainsi un motif éthique qui lui permet de sortir du dilemme devant lequel le projet de Pharnace la placerait. La réponse de Pharnace ne se fait pas directement, au niveau de l'argument lui-même. Elle dénonce d'emblée le procédé indirect de Monime et y répond par l'indirection :

Que parlez-vous de Rome et de son alliance?

Pourquoi tout ce discours et cette défiance?

Qui vous dit qu'avec eux je prétends m'allier? [...]

De mes intentions je pourrais vous instruire,

Et je sais les raisons que j'aurais à vous dire,

Si laissant en effet les vains déguisements

Vous m'aviez expliqué vos secrets sentiments.

Mais enfin je commence, après tant de traverses,

Madame, à rassembler vos excuses diverses ;

Je crois voir l'intérêt que vous voulez celer,

Et qu'un autre qu'un père ici vous fait parler. [Mithridate, v. 275-290]

Le déploiement rhétorique est perçu comme un écran de parole devant l'« intérêt » caché et un énonciateur caché derrière l'oratrice elle-même. L'intuition désarme la logique et anticipe la reconnaissance du rival Xipharès. Ce dernier reprend le mot central dans la réplique de son frère ( $Q$ Quel que soit l'intérêt qui fait parler la reine ", v. 291) pour tenter de retourner à l'argumentation, de ramener la scène sur le terrain politique. Il cherche à empêcher que la scène politique ne tourne en scène de rivalité amoureuse [v.311-314]: "Vivons ou périssons dignes de Mithridate, / Et songeons bien plutôt, quelque amour qui nous flatte, / À défendre du joug et nous et nos États / Qu'à contraindre des cœurs qui ne se donnent pas. » Mais la stratégie demeure inefficace puisque Pharnace traverse encore le discours politique pour retrouver le discours amoureux et procéder à une opération de prédication, fruit d'une déduction purement intuitive, qui donne à l'imprécis "intérêt » un contenu défini et aux fantômes argumentatifs une présence actuelle [v. 315-317] : « Il sait vos sentiments. Me trompais-je, Madame ? / Voilà cet intérêt si puissant sur votre âme, / Ce père, ces Romains que vous me reprochez. » Face à la pénétration lucide de Pharnace, la feinte de Xipharès apparaît d'autant plus comme une défaite et le conditionnel comme un renoncement à l'effort persuasif même [v. 318-320]: "J'ignore de son cœur les sentiments cachés; / Mais je m'y soumettrais sans vouloir rien prétendre / Si, comme vous, Seigneur, je croyais les entendre. » La fidélité aux secrets trahis, que revendique Pharnace, dans le pacte de non-agression qu'il propose à son frère, étend sur la scène une vaste chape de dissimulation destinée à faire pièce aux « trompeuses adresses » de 
Mithridate dont l'art est, précisément, dit Pharnace vers 370, de « s'arme[r] contre nous de nos moindres discours. » Cette relation discursive est emblématique à de nombreux égards du traitement de l'argumentation dans nos pièces : les personnages retournent l'arme de la persuasion contre l'orateur qui a cherché à la produire et la désamorcent avant même qu'elle puisse agir. Sur scène prévaut en effet la dimension herméneutique de la rhétorique contre sa dimension proprement argumentative.

\section{Une rhétorique herméneutique}

L'auditoire intrascénique prend en défaut l'orateur auquel manque le consilium, qui, selon Quintilien, permet à l'orateur de «se retourner selon les exigences variées de la conjoncture ${ }^{19}$ ", de s'adapter " aux lieux, aux temps, aux personnes ». Dans un premier exemple, en Britannicus, II, 3, Néron se désigne comme le prétendant de Junie et justifie son « choix » de l'époux de la princesse, ce qui, en soi, représente un premier sophisme puisqu'il s'institue ainsi arbitrairement instance décisionnaire pour autrui. L'empereur utilise le moment argumentatif pour achever de parfaire son identité en devenant l'objet même de la décision qui lui confère son autonomie : Néron, dans une structure simultanément double ${ }^{20}$ et circulaire relevant de la faute logique, s'affirme à la fois origine et terme $\mathrm{du}$ choix, sujet et objet du choix. Sa décision, parfaitement autonomisante, est manifeste dans l'enchaînement qui mène à sa verbalisation :

Ma mère a ses desseins, Madame, et j'ai les miens.

Ne parlons plus ici de Claude et d'Agrippine :

Ce n'est point par leur choix que je me détermine.

C'est à moi seul, Madame, à répondre de vous,

Et je veux de ma main vous choisir un époux. [Britannicus, v. 562-565]

13 Avant d'en arriver à l'énoncé laconique de l'objet du choix [v. 572] : "Moi, madame », Néron l'a justifié sur une trentaine de vers où domine l'argument de la cohérence, de la plénitude, de l'accomplissement : le choix se trouve même hyperboliquement présenté comme convenant et bienséant [v. 602], «Digne de l'univers à qui vous vous devez ». Mais Junie, confrontée également à l'argument sophistique selon lequel elle serait coupable d'avoir "relégué » l'empereur « dans sa cour ${ }^{21}$ ", dénonce l'aspect contourné du raisonnement et la perversion de l'ordre du vraisemblable. Pour elle, le discours de Néron, les arguments qu'il utilise, entrent en contradiction avec les circonstances réelles sur lesquelles ce discours entend agir : en II, 3 [v. 603 sq.] elle manifeste donc son « étonnement » devant la témérité avec laquelle elle se voit « dans le cours d'une même journée, / Comme une criminelle amenée en ces lieux » pour se voir ensuite offrir «tout d'un coup la place d'Octavie.» La prétention de Néron à faire de son intérêt particulier le critère de la vraisemblance le conduit au contraire à priver le discours de toute pertinence et de toute propriété. Alors que Junie exprime son refus d'entrer en rivalité avec Octavie, Néron, comme Pharnace en exposition de Mithridate face à Monime, voyant se dévoiler la logique de son propre intérêt, en vient à traduire l'intérêt implicite au discours de Junie [v.636-637]: « Mais ne nous flattons point, et laissons le mystère : / La sœur vous touche ici beaucoup moins que le frère, / Et pour Britannicus...» Il est frappant ici que Néron, malgré son défaut de prudence rhétorique $^{22}$, déplie avec pertinence le discours de Junie. Les personnages font en effet davantage preuve de discernement et de jugement lorsqu'ils déploient la traduction du discours dont ils sont la cible. La distance leur permet la lecture rhétorique dont ils ne sont pas capables dans leur propre adresse à l'autre. Les héros s'interrogent ainsi avec 
insistance sur la vraisemblance des discours qu'on développe devant eux et à leur destination. Alors que l'orateur doit savoir saisir les signes qui révèlent la disposition de son auditoire, les héros se représentent en tant qu'auditeurs et s'attachent à saisir les signes qui révèlent la disposition de l'orateur qui leur fait face. C'est à un schéma persuasif en quelque sorte inversé que nous avons affaire en ce sens que la recherche du discours vraisemblable, sa mise en évidence et en spectacle, fait moins l'objet d'un travail précédant le discours persuasif que d'une réception de ce discours persuasif. Le pôle rhétorique critique, et donc dynamique, est celui du récepteur du discours. La réflexion rhétorique sert moins la persuasion que la mise en évidence de sa mise en échec. La lecture rhétorique est alors dramatiquement opérante: ainsi Burrhus, en III, 2, démystifie la fascination manifestée par Agrippine à l'égard de son propre discours déréglé ; alors que la mère de Néron se projette avec impétuosité dans un discours auto-accusateur devant l'armée instituée en témoin et en juge ${ }^{23}$ de ses propres forfaits, le gouverneur, en vertu même d'une lucidité technique, l'interrompt et la dénonce comme invraisemblable [v. 855-856]: «Madame, ils ne vous croiront pas. / Ils sauront récuser l'injuste stratagème / D'un témoin irrité qui s'accuse luimême. »

14 Ainsi, ce n'est pas l'orateur qui manifeste avec le plus d'éclat la connaissance morale sur laquelle fonder l'entreprise de persuasion mais l'auditeur qui repère chez son interlocuteur les passions comme, selon les termes d'Aristote, «les causes qui font varier les hommes dans leurs jugements ${ }^{24} »$. C'est l'auditeur qui repère la présence du pathos chez l'orateur et cherche à le rationaliser, à montrer l'impertinence d'un ethos perturbé ou instable, d'une maitrise oratoire insuffisante, d'une inconvenance condamnant le discours à l'invraisemblance. À de nombreuses reprises, plus l'orateur présente son discours comme nécessaire et plus son destinataire le reçoit comme contingent, dénonçant chez le premier une propension téméraire à prendre pour certaines des preuves seulement probables. Dans cette paraphrase interprétative, le destinataire opère un décrochement immédiat de l'attention vers les prémisses, pratiquant ainsi ce que l'on pourrait appeler une «rhétorique de l'intention " pour réfuter la communauté de ces prémisses entre l'orateur et son auditeur et donc tout accord préalable sur lequel pourrait ensuite se fonder l'effort de persuasion. L'opérateur régulier de ce décrochement se reconnaît dans la formule «Je vous entends». Cette formule trouve sa préfiguration dans l'impératif de Jocaste à Créon en I, 5 [v. 223-26] : « Mais avouez, Créon, que toute votre peine / C'est de voir que la paix rend votre attente vaine, / Qu'elle assure à mes fils le trône où vous tendez, / Et va rompre le piège où vous les attendez "; et dans les assertions symétriques de Créon et d'Antigone à propos d'Hémon [v. 273-282]: «Je sais ce qui le rend innocent à vos yeux » / Antigone : «Et je sais quel sujet vous le rend odieux. »; «Et l'amour du pays nous cache une autre flamme. / Je la sais ; mais, Créon, j'en abhorre le cours, / Et vous ferez bien mieux de la cacher toujours. » Le savoir exclut la réception primaire de l'argument: il la surdétermine immédiatement. L'exposition de Britannicus fait entendre ce décrochement à l'égard de la situation dialectique : Agrippine procède à la déduction des prémisses cachées du discours de Burrhus en introduisant dans leur échange le tiers invisible, Néron, invalidant le contenu du discours du gouverneur, simple écran devant le discours implicite de l'empereur perçu comme stratégique :

Je vous entends : Néron m'apprend par votre voix

Qu'en vain Britannicus s'assure sur mon choix.

En vain, pour détourner ses yeux de sa misère, 
J'ai flatté son amour d'un hymen qu'il espère.

À ma confusion, Néron veut faire voir

Qu'Agrippine promet par delà son pouvoir.

Rome de ma faveur est trop préoccupée :

Il veut par cet affront qu'elle soit détrompée,

Et que tout l'univers apprenne avec terreur

À ne confondre plus mon fils et l'empereur. [Britannicus, I, 2, v. 245-254]

Quand il s'agit, en III, 7, de lever le voile sur l'invisible présence de Néron en II, 6, le jeu ironique de Racine sur le verbe « entendre » manifeste cette fois le tragique défaut de la lucidité de Britannicus devant la prudence de Junie [v. 957] : «Retirez-vous, Seigneur, et fuyez un courroux / Que ma persévérance allume contre vous. » Lorsque Britannicus dit « entendre " Junie (v. 964-966: «Je vous entends, Madame, / Vous voulez que ma fuite assure vos désirs, / Que je laisse un champ libre à vos nouveaux soupirs. ») il lui "impute ${ }^{25}$ " de fait des sentiments que sa seule jalousie d'amant projette. Néron, en III, 8, utilisera la même cheville laconique pour économiser tout échange argumenté avec son rival. Cultivant le détour, notamment dans l'énallage des pronoms personnels qui évite alors l'interlocution directe au début de l'échange, la joute doit faire naitre la terreur chez le spectateur. Car l'audace verbale de Britannicus entre en contradiction brutale avec l'infériorité de son statut et les répliques progressent vers une provocation frappée de gratuité : «Je ne sais pas du moins épier ses discours. / Je la laisse expliquer sur tout ce qui me touche, / Et ne me cache point pour lui fermer la bouche ». La réponse de Néron donne à l'action le pouvoir de combler l'ellipse argumentative [v. 1066-1069] : «Je vous entends. Eh bien, gardes! ». Le « je t'entends » de Mithridate s'oppose quant à lui, avec une variabilité inquiétante, tout autant au refus de Monime d'épouser Pharnace, dans une déduction entravée par la prévention (voir III, 5, v. 1079-1095 : «Je le vois bien : quelque effort que je fasse, / Madame, vous voulez vous garder à Pharnace. »; " Vous résistez en vain, et j'entends votre fuite... »), qu'au refus argumenté de Pharnace de suivre l'armée de son père dans sa conquête de Rome, dans une déduction cette fois clairvoyante [III, 1, v. 970-971]: "Tu ne saurais partir, perfide, et je t'entends. / Je sais pourquoi tu fuis l'hymen où je t'envoie [...]. » La formule sanctionne à chaque reprise un renversement argumentatif de la valeur des discours ${ }^{26}$ qui rend la persuasion impossible.

Cet effort herméneutique, opposé à l'effort rhétorique comme effort persuasif, conduit à contourner la dialectique : quand on argumente sur le procédé, on n'argumente plus sur le fond de la thèse proposée et la rhétorique perd de son pouvoir heuristique, par lequel on peut éclairer un choix, le plus vraisemblable, pour être détournée à des fins herméneutiques, par lesquelles on cherche à se ménager un pouvoir de domination et donc d'action sur l'autre. La " sophistique de la ruse ${ }^{27}$ ", pour reprendre une expression de G. Declercq, n'est pas seulement effort pour tromper l'autre, elle est surtout volonté de le connaître, ambition de le transpercer, et donc de le maîtriser. G. Declercq insiste sur la distance instituée par Racine entre le spectateur et «la rhétoricité du discours intrascénique», distance autorisant la "conversion esthétique du discours argumentatif intrascénique ", le spectateur retirant son plaisir des fautes oratoires des personnages. Il nous semble possible de proposer que cette distance est réduite par le dédoublement d'une rhétoricité prise en charge par les personnages eux-mêmes. La "rhétorique du dramaturge à l'adresse du spectateur ${ }^{28}$ " n'exclut pas les personnages de la compétence métarhétorique qui serait dévolue à ce seul spectateur: les ruses oratoires n'échappent pas aux personnages et leurs efforts de paraphrase persuasive 
participent également du plaisir de la lucidité offert au spectateur. Ce dédoublement apparaît comme une des conditions de l'animation dramatique de l'argumentation ${ }^{29}$, l'un des moyens qui permet de "plaire et toucher ", "invente[r] des ressorts " qui puissent "attacher ", et éviter les "froids raisonnements" autant que les "vains efforts" de la "rhétorique", comme le recommande Boileau au chant III de l' Art poétique. La réunion, selon les termes de J. Morel, d'une rhétorique explicite et seconde s'adressant au spectateur par l'intermédiaire d'un interlocuteur sur scène et d'une rhétorique implicite et primaire s'adressant au spectateur, se produit d'autant plus efficacement que le dédoublement de la rhétorique en sa dimension persuasive et en sa dimension herméneutique est déjà le fait des personnages eux-mêmes lorsqu'ils cherchent à opérer la traversée de l'argumentation dont ils sont la cible. L'objet de la mise en scène devient la crédibilité elle-même : la rhétorique, de technique structurant le discours, devient objet de représentation, la mise en abyme de son propre déchiffrement participant de la structuration de l'action dramatique.

\section{L'effet rhétorique}

La récurrente interrogation de la crédibilité des discours par la prudence examinatrice des personnages permet à Racine d'opérer un renversement notable, privant l'orateur de cette prudence et l'accordant aux auditeurs. C'est de cette mise en scène de la crédibilité que le rapport de force entre Britannicus et Narcisse tire sa puissance pathétique. Britannicus, désorienté par la dissimulation de Junie qu'il a prise pour une trahison en II, 6, ne remet pas en doute la fidélité de Narcisse alors qu'il « fonde son assurance » [III, 6, v. 928] sur la parole du traître pour soupçonner celle de Junie. Face au confident qui manipule sa créance et l'interroge sur l'efficacité persuasive d'une parole dont Racine a fait délibérément l'ellipse sur la scène [v. 935] : «Après tous mes discours, vous la croyez fidèle? ", Britannicus cède à l'invraisemblance promue au rang de vérité et pourtant mêlée d'hésitation :

Non, je la crois, Narcisse, ingrate, criminelle,

Digne de mon courroux. Mais je sens, malgré moi,

Que je ne le crois pas autant que je le dois.

Dans ses égarements mon cœur opiniâtre

Lui prête des raisons, l'excuse, l'idolâtre.

Je voudrais vaincre enfin mon incrédulité,

Je la voudrais haïr avec tranquillité.

Et qui croira qu'un cœur si grand en apparence,

D'une infidèle cour ennemi dès l'enfance,

Renonce à tant de gloire, et dès le premier jour

Trame une perfidie, inouïe à la cour ? [Britannicus, v. 936-346]

Britannicus a perçu l'incohérence entre le caractère de Junie et le comportement qui doit être déduit de son discours. Il énonce, avec son "incrédulité », l'absence de nécessité doxale de la persuasion dont il est l'objet; le discours de Junie n'est pas vraisemblable : il faut donc qu'une autre nécessité, extérieure à la relation rhétorique, se substitue à la vraisemblance attendue. C'est dans ce cadre que la duplicité de Narcisse soutient la plus grande ironie tragique: le fallacieux confident conseillait en I, 4, à sa victime le discernement et la prudence. Ce faisant, il affichait un attachement à la droiture qui construisait le piège de la véracité oratrice par rapport auquel l'authenticité de Junie se trouve dégradée et comme renversée. Les mots de Britannicus sonnent ainsi comme des antiphrases emblématiques de ce renversement 
des valeurs [I, 4, v. 339-342] : «Narcisse, tu dis vrai. Mais cette défiance / Est toujours d'un grand cœur la dernière science,/ On le trompe longtemps. Mais enfin je te crois. / Ou plutôt je fais vœu de ne croire que toi. » Au nom de sa sincérité et de son intégrité morale, valeurs qui sont au cœur de son partage avec Junie, Britannicus excuse son défaut de lucidité et énonce une vérité dramatique sans le savoir puisqu'il est, au moment même où il voudrait ne pas l'être, crédule, c'est-à-dire, persuadé à tort. Le pathétique de la situation vient de la confusion qui s'opère en Britannicus entre les pressions respectives d'un devoir imaginaire et de sa passion : dupé par la situation mise en scène par Néron, il croira croire Narcisse par devoir et contre sa passion, alors qu'il le croit par aveuglement et contre ses devoirs envers Junie, contre toutes les lois de la vraisemblance.

Cette profession d'aveuglement de Britannicus entre en résonance avec le renoncement de Monime acculée à l'aveu par la pression actrice, proprement hypocrite, de Mithridate, cédant à une feinte renversée en vraisemblance par son indignité, son inconvenance même [III, 5, v. 1096-1097]: «En quelle extrémité, Seigneur suis-je réduite? / Mais enfin je vous crois, et je ne puis penser / Qu'à feindre si longtemps vous puissiez vous forcer. » Mais l'aveu à peine formulé, c'est une didascalie interne qui signale le retour à la vérité et la récusation d'une construction vraisemblable abusive [v.1111-1112]: «Avant que votre amour m'eût envoyé ce gage, / Nous nous aimions... Seigneur, vous changez de visage !». La perplexité douloureuse qui s'ensuit (v. 1116: «O ciel! me serais-je abusée?») est propre à signifier cette connivence pénible entre la vraisemblance et la fausseté, manifestant l'instrumentalisation de la relation rhétorique à des fins de pouvoir. La situation est si grave d'enjeux pour le poète tragique qu'il la prolonge dans la confrontation avec la confidente, Phœdime, qui, face à la terreur de Monime devant sa crédulité (IV, 1, v. 1134-1135 : « Mais il feignait peut-être ? Il fallait tout nier. / Le roi feignait ! Et moi, découvrant ma pensée... »), reconstruit la crédibilité du discours du roi, fondée sur son ethos même de roi [v. 1147-1149]: «Ah! traitez-le, Madame, avec plus de justice:/ Un grand roi descend-il jusqu'à cet artifice? / À prendre ce détour qui l'aurait pu forcer ? » L'effort de persuasion, de fait falsifié, est mis en scène dans sa fragilité : Monime "cède aux raisons » de sa confidente " autant qu'elle le peut » [v.1166-1168] tout en confrontant cette vraisemblance invoquée à la vérité de la situation qui voit tarder la venue de Xipharès. Cette proximité de la vraisemblance avec la fausseté, son instrumentalisation par la feinte, sert la représentation de l'argumentation comme aperçu donné sur la fausseté intellectuelle et la noirceur morale. Fondée sur des prémisses fabriquées en vue d'une fin à atteindre, les discours persuasifs de Narcisse et de Mithridate, dans les situations que nous venons d'examiner, constituent des artefacts de vraisemblance et n'ont aucune prétention référentielle. Aussi la praxis affiche-t-elle son mépris de toute consistance ontologique des arguments.

Lorsque Néron ordonne à Junie de prononcer un discours disconvenant à l'égard de son caractère comme à l'égard de la situation en présence ${ }^{30}$, Junie ne manque pas de souligner cette disconvenance [II, 3, v. 675-677] : « Moi ! Que je lui prononce un Arrêt si sévère. / Ma bouche mille fois lui jura le contraire. » L'effet sur le spectateur est très puissant : ce dernier attend de voir se déployer une relation rhétorique condamnée à l'avance, faussée d'emblée. La conduite du raisonnement de Néron elle-même a été dénoncée comme invraisemblable : Junie a remis ainsi en cause la capacité à établir une intelligence de la situation commune à l'orateur, elle-même, et à son auditoire, 
Britannicus, à tisser ce lien doxal duquel dépend la persuasion. Lorsqu'en IV, 4, Mithridate vient chercher Monime pour son mariage après lui avoir présenté le leurre d'une union avec Xipharès, Monime résiste en feignant une fausse évidence s'appuyant sur les arguments fallacieux utilisés par le roi en III, 5 (v. 1278 : « Et ne m'avez-vous pas défendu d'y penser?»), feinte qui a pour conséquence immédiate de dénoncer l'instrumentalisation stratégique de l'argumentation préalable: "J'eus mes raisons alors; oublions-les, Madame...»[v. 1279]/ «Quoi, Seigneur! vous m'auriez donc trompée...» [v. 1284]. Monime porte alors son propre effort persuasif pour dénoncer désormais la ruine de toute confiance, de tout lien doxal à venir :

Vos détours l'ont surpris, et m'en ont convaincue.

Je vous l'ai confessé, je le dois soutenir.

En vain vous en pourriez perdre le souvenir,

Et cet aveu honteux, où vous m'avez forcée,

Demeurera toujours présent à ma pensée.

Toujours je vous croirais incertain de ma foi ${ }^{31}$.

21 Pour son destinataire, le défaut de justice affecte le discours et suspend sa valeur persuasive: les personnages dénoncent alors une «injuste prière» (Thébaïde , II, 3, v. 528), « l'injuste stratagème » (Britannicus, III, 2, v. 856), ou d'« injustes mépris ", (Mithridate, III, 5, v. 1081), qui trahissent la faiblesse du discours par lequel l'orateur entend rallier son interlocuteur ${ }^{32}$. C'est encore une fois l'effet spectaculaire de l'argumentation qui prime sur son effet persuasif. Au moment de contracter une alliance avec Britannicus, Agrippine, malgré les menaces qui s'accumulent, se figure encore, dans un tableau fantasmatique, en oratrice :

Le coupable Néron fuit en vain ma colère :

Tôt ou tard il faudra qu'il entende sa mère.

J'essaierai tour à tour la force et la douceur,

Ou moi-même, avec moi conduisant votre sœur,

J'irai semer partout ma crainte et ses alarmes,

Et ranger tous les cœurs du parti de ses larmes.

Adieu. J'assiégerai Néron de toutes parts. [Britannicus, III, 5, v. 919-925]

L'image du siège inscrit le procès rhétorique dans le registre militaire et fait prévaloir de manière spectaculaire sa dimension agonistique. L'adhésion recherchée est une adhésion au spectacle que donne le discours persuasif tandis que seule la distance peut s'instaurer avec l'argumentation en passe d'être développée devant le spectateur. L'expression choisie par Monime, après l'aveu de son amour partagé, au moment d'exiger de Xipharès à la fois son départ et un effort démonstratif visant à justifier ce départ auprès de Mithridate, est très éloquente :

Inventez des raisons qui puissent l'éblouir.

D'un héros tel que vous c'est là l'effort suprême :

Cherchez, Prince, cherchez, pour vous trahir vous-même,

Tout ce que, pour jouir de leurs contentements,

L'amour fait inventer aux vulgaires amants. [Mithridate, II, 6, v. 722-726]

L'effort persuasif consiste à construire une argumentation fictive où les preuves intrinsèques se soutiennent de leur propre prolifération; l'évidence de sa nécessité pragmatique rend l'exposé de sa nécessité logique superflue [v. 703-706] : «J'attends du moins, j'attends de votre complaisance/Que désormais partout vous fuirez ma présence. / J'en viens de dire assez pour vous persuader / Que j'ai trop de raisons de vous le commander. » L'hyperbole suffit à exprimer la nécessité de ces preuves dont l'exposé est suspendu. Dans Britannicus, en IV, 2, un autre type d'hyperbole, confinant à 
l'abus rhétorique, conduit le dialogue entre Néron et Agrippine à tourner court. Néron semble céder à la pression argumentative, à haute charge affective, du discours grandiloquent de sa mère [v. 1285] : « Eh bien donc! prononcez. Que voulez-vous qu'on fasse? "; il ménage une illusion de victoire à Agrippine [v. 1302] : "Je vous fais notre arbitre, et vous nous jugerez.» La suite montre pourtant que, là encore, il s'agit d'économiser l'effort rhétorique et d'opposer l'action à l'argumentation.

La nécessité d'agir impose en effet un autre type d'économie rhétorique en conduisant le personnage à se détourner de la recherche des preuves, de l'établissement raisonné d'une persuasion par le discours, pour privilégier la confrontation scénique aux faits et non aux arguments. L'enchaînement des scènes 3 et 4 de l'acte III de Mithridate, liées par la récurrence, en écho, du verbe " croire » (le verbe apparaît sept fois dans les deux scènes), à la suite de la dénonciation de Xipharès par Pharnace, place à nouveau sous le regard du spectateur le travail d'un personnage sur sa conviction, travail qui met en jeu le discernement et la prudence du personnage. À la scène 3, Xipharès en appelle à l'évidence de son propre ethos: "Seigneur, le croirez-vous, qu'un dessein si coupable... ». Mithridate répond cette fois de manière directe et pertinente :

Mon fils, je sais de quoi votre frère est capable.

Me préserve le ciel de soupçonner jamais

Que d'un prix si cruel vous payez mes bienfaits;

Qu'un fils qui fut toujours le bonheur de ma vie

Ait pu percer ce cœur qu'un père lui confie!

Je ne le croirai point. [Mithridate, v. 999-1005]

Or, cette mise en place d'un accord rhétorique est immédiatement dénoncée comme une feinte: l'évidence éthique sur laquelle Xipharès fonde l'invraisemblance de l'accusation de Pharnace va s'avérer pour Mithridate suspecte de manipulation irrationnelle, il va même jusqu'à la soupçonner de n'être qu'une fiction passionnelle. Mithridate interrompt le discours de son fils et fait avorter son argumentation; sa réplique, construite de manière à rendre l'argumentation inutile, est destinée en fait à la différer; le roi entend décider seul. L'affirmation catégoriquement laconique qui entend clore le questionnement de Mithridate: "Je ne le croirai point", est une réponse imposée à une question interdite : la structure même de l'échange est tendue vers une forme d'écrasement par le père. La situation tragique met notamment ici en relief un jeu délibéré du dramaturge avec l'examen de l'ethos, du caractère de l'orateur qui, en principe, guide le jugement de celui qui doit donner sa foi. Le renversement entre la scène 3 et la scène 4 montre Mithridate accorder sa confiance à Xipharès sur la base d'un jugement vraisemblable, puis la lui retirer en dégradant sa propre confiance en son propre jugement lui-même jugé impondéré. La délibération monologuée de la scène 4 est marquée par un renversement formel reflet du renversement psychologique du personnage de la méfiance à la confiance, puis au doute et enfin à la décision, qui manifeste le renoncement à la confiance, et le choix d'une pleine autonomie de la décision déliée de toute persuasion extérieure. Le monologue, procédé controversé chez les théoriciens pour son caractère artificiel, trouve ici sa pleine justification du fait de la volonté de Mithridate de se libérer de l'effort persuasif de Xipharès ${ }^{33}$. Le personnage entend se soustraire à l'aliénation à l'autre mais envisage aussi le risque de l'aliénation à soi-même dans l'illusion [v. 1008 sq.] : «Je ne le croirai point? Vain espoir qui me flatte! / Tu ne le crois que trop, malheureux Mithridate! » Le questionnement porte précisément encore sur la crédulité et l'incrédulité et non plus sur la persuasion. Le monologue traduit donc exactement, et formellement, la situation dramatique et 
psychologique dans laquelle se trouve le personnage et qui s'exprime par un tour généralisant accentuant la déshumanisation du contexte de la parole, son abstraction à l'égard de toute relation à l'autre. La totalité est ainsi renvoyée du côté de l'absence, paradoxalement : "Tout m'abandonne ailleurs ? Tout me trahit ici ? / Pharnace, Amis, Maîtresse ? Et toi, mon Fils, aussi ?» L'aposiopèse marque l'acmé du pathétique : "Toi qui de la vertu consolant ma disgrâce... »; puis se produit un ressaisissement rationnel marqué par le retour à la fiabilité de la connaissance passée de l'ethos du «perfide Pharnace » et la connaissance présente de son pathos ; l'argument de l'identité («Mais ne connais-je pas le perfide Pharnace ?») et celui de la passion du personnage (v. 1015-1020 : "Quelle faiblesse à moi d'en croire un Furieux / Qu'arme contre son Frère un dessein envieux, / Ou dont le désespoir me troublant par des fables, / Grossit, pour se sauver, le nombre des coupables!») sont convoqués pour servir un discernement dont le roi voudrait s'assurer. La progression de la délibération est alors très rapide et aboutit au choix d'un "artifice». Le "choix" s'exprime en des vers extrêmement sobres, presque prosaïques [v. 1025-1027] : «Qu'on appelle la Reine. Oui, sans aller plus loin,/Je veux l'oür. Mon choix s'arrête à ce témoin./L'amour avidement croit tout ce qui le flatte. » Le déplacement du verbe "croire » est alors remarquable: il se trouve désormais détaché du personnage de Mithridate pour être transféré sur Monime. Le risque de la crédulité est mis à distance et déplacé vers le personnage féminin qu'il s'agit de soumettre à l'ambiguïté pour qu'elle la lève en tombant dans le piège de la crédulité, précisément. Le coupable recherché depuis la scène 3, l'ingrat refusé en Xipharès et trop connu en Pharnace, trouvent leur projection dans le personnage de Monime désignée par un seul adjectif substantivé censé résumer son ethos présent [v. 1028] : "Qui peut de son vainqueur mieux parler que l'ingrate?». La sortie du monologue se trouve donc spatialisée par l'arrivée de la reine (« Mais je la vois paraître ») qui signe le retour au dialogue et à l'action par-delà tout effort délibératif et même persuasif puisque l'effort argumentatif qui va suivre ne sera qu'une «feinte » cherchant précisément à entraver tout raisonnement vraisemblable chez Monime (v. 1033-1034 : « Feignons, et de son cœur, d'un vain espoir flatté, / Par un mensonge adroit tirons la vérité.») pour privilégier la mise au jour d'une preuve extrinsèque irréfutable. C'est à Monime qu'est déléguée, indirectement et hors du cadre $\mathrm{du}$ discours judiciaire, la fonction de juge [v. 1029] : «Voyons, qui son amour accusera des deux. " L'issue du monologue est permise par une extériorisation de la difficulté herméneutique, une projection du doute chez l'autre, chargée, en l'absence de délibération efficace, de lever le doute du juge lui-même. Mithridate ne s'expose plus ainsi à se trouver au centre d'une ironie tragique mordante lorsqu'il déléguait par erreur à Xipharès le soin de détourner Monime de son faux amant Pharnace en arguant de sa neutralité :

Vous cependant ici veillez pour mon repos:

D'un rival insolent arrêtez les complots,

Ne quittez point la reine, et s'il se peut, vous-même

Rendez-la moins contraire aux vœux d'un roi qui l'aime.

Détournez-la, mon fils, d'un choix injurieux;

Juge sans intérêt, vous la convaincrez mieux ${ }^{34}$.

26 C'est donc sur le statut même de l'argumentation que la tragédie conduit à s'interroger. Ironiquement identifiée à l'action au v. 556 de La Thébaïde: "Laissez agir encor la princesse et la reine » (" agir » étant ici un synonyme d'« argumenter»), elle se trouve dénoncée comme une manœuvre sophistique ayant effectivement conduit l'action tragique $^{35}$. Les images choisies dans nos pièces pour représenter l'argumentation 
l'opposent à la pensée, au savoir, à l'échange. Elles interrogent ainsi son rapport à la vérité et à sa dissimulation ${ }^{36}$. La mise en crise de la vraisemblance sur scène et l'effort intrascénique pour la discerner ne sont pourtant pas les symptômes d'un renoncement à la rationalité rhétorique mais celui d'un dysfonctionnement de celle-ci, employée comme mécanisme de défense contre toute persuasion, toute construction à prétention vraisemblable, la rhétorique, dans sa dimension argumentative, étant perçue, dans le cadre du conflit tragique, comme une prétention de l'autre au pouvoir sur soi, comme une aliénation. Dans nos pièces, la rhétorique réussit comme technique de connaissance mieux que comme technique de persuasion: elle entre ainsi avec succès dans la composition des discours qui portent le "caractère Théâtral» et dans l'animation d'un univers tragique représenté comme un univers d'herméneutes aveugles dès qu'il s'agit de persuader mais puissants à perpétuer le conflit.

\section{NOTES}

1. Éd. H. Baby, Paris, Champion, 2001, p. 430.

2. Ibid., p. 431.

3. Sur les rapports de Racine à la rhétorique voir G. Declercq, «La formation rhétorique de Racine ", Jean Racine, 1699-1999, Actes du colloque du tricentenaire (25-30 mai 1999), éd. G. Declercq et M. Rosellini, Paris, PUF, 2003, p. 257-290. Voir aussi l'article de M. Hawcroft, «La mauvaise rhétorique chez Racine», La Licorne, 50,1999, p. 355-368 qui recense certains préceptes recopiés par Racine chez Quintilien.

4. Réflexions sur la poétique d'Aristote, Paris, François Muguet, 1674, II, XXI, p. 106-107; cité par J. Lecompte, Raison et vraisemblance à l'âge classique. Statut de la rhétorique chez René Rapin et Jean Racine, 2007, Paris III, p. 408.

5. Sur cette question, voir en particulier A. Kibedi Varga, Rhétorique et littérature. Études de structures classiques [1970], Paris, Klincksieck, 2002, p. 96.

6. Pratique du théâtre, op. cit., p. 407-408.

7. Rhétorique, livre I, chap. II, I, trad. C.-E. Ruelle revue par P. Vanhemelryck, Paris, Le Livre de Poche, 1991, p. 82.

8. Toutes nos références au texte de Racine se feront dans l'édition J. Morel et A. Viala, Paris, Classiques Garnier, 2010 ; I, 2, v. 37-42.

9. «Poéticité versus rhétoricité : pathos et logos dans les tragédies de Racine », Racine et / ou le classicisme, Biblio 17, 129,2001, p. 48. P. France montre que règne chez Racine une "rhétorique fonctionnelle " prenant le dessus sur une rhétorique théâtrale "décorative ", Racine's Rhetoric, Oxford, 1965 ; voir aussi sur ce point D. Maskell, Racine. A Theatrical Reading, Oxford, Clarendon Press, 1991.

10. G. Declercq évoque « la beauté d'un échec oratoire répété » et voit « la formule racinienne de la catharsis " dans "cette conversion d'un échec rhétorique intrascénique en accomplissement esthétique extrascénique ", " Poéticité versus rhétoricité : pathos et logos dans les tragédies de Racine ", op. cit., p. 50.

11. Institution oratoire, VI, 2, 28.

12. Par exemple Mithridate, II, 5, v. 607-608: «Un fils audacieux insulte à ma ruine, / Traverse mes desseins, m'outrage, m'assassine » 
13. La Thébaïde, IV, 3, v. 1181, par exemple.

14. IV, 1, v. 905-906 : Étéocle désavoue à l'avance l'argumentation en cours : « Nous verrons ce qu'il veut; mais je répondrais bien / Que par cette entrevue on n'avancera rien ». "Qu'auprès du diadème il n'est rien qui vous touche. ", "N'accorderez-vous rien aux larmes d'une mère?", « Hélas! rien ne les touche!»

15. Par exemple, Mithridate, IV, 2, v. 1261-1263, Monime à Xipharès : "Allez; de ses fureurs songez à vous garder, / Sans perdre ici le temps à me persuader : / Le ciel m'inspirera quel parti je dois prendre."

16. Par exemple Néron face à Junie en Britannicus, II, 3, v. 636-637 : « Mais ne nous flattons point, et laissons le mystère:/ La sœur vous touche ici beaucoup moins que le frère, / Et pour Britannicus... »

17. M. Hawcroft a analysé, dans la même perspective, l'extravagance rhétorique à laquelle cède Agrippine en IV, 1 en méprisant les conseils de Burrhus qui, préparant son entrevue avec Néron, l'oriente vers une situation judiciaire où elle pourra "se défendre" mais non pas accuser l'empereur, puis en IV, 2 , en entrant en contradiction manifeste avec cette mise en garde, dans un discours maladroit et inversant irrésistiblement la défense en accusation comme le montre la péroraison: "Et lorsque, convaincu de tant de perfidies, / Vous deviez ne me voir que pour les expier, / C'est vous qui m'ordonnez de me justifier» (v.1220-1222). Comme Agrippine, Mithridate cherchera, face à Monime, en IV, 4 , à inverser une situation judiciaire dont il dénonce l'impertinence : "Cependant quand je veux oublier cet outrage, / Et cacher à mon cœur cette funeste image, / Vous osez à mes yeux rappeler le passé ; / Vous m'accusez encore, quand je suis offensé ! » (v. 1307-1310).

18. Par exemple, dans La Thébaïde, en I, 5 où les assertions se succèdent en prenant la forme de reformulations de l'argument précédent: «Est-ce au peuple, Madame, à se choisir un maître? " / "Que le peuple a son gré nous craigne ou nous chérisse, / Le sang nous met au trône, et non pas son caprice. » La correction argumentative affecte également dans cette scène la prédication du nom de «tyran ». Voir aussi la scène II, 3, où la joute entre Antigone et Polynice se poursuit au gré de corrections successives sur la prédication attachée au verbe « aimer » : «Estce m'aimer, cruel, autant que je vous aime, / Que d'être inexorable à mes tristes soupirs, / Et m'exposer encore à tant de déplaisirs?" — «Mais vous-même, ma sœur, est-ce aimer votre frère / Que de lui faire ici cette injuste prière, / Et me vouloir ravir le sceptre de la main?» (v. 524 sq.).

19. Institution oratoire, II, $13,2$.

20. Le personnage se dédouble en sujet et objet aux v. 579-582: «Plus je vois que César, digne seul de vous plaire, / En doit être lui seul l'heureux dépositaire, / Et ne peut dignement vous confier qu'aux mains / À qui Rome a commis l'empire des humains. »

21. V.545-546: "Pourquoi, de cette gloire exclu jusqu'à ce jour,/M'avez-vous, sans pitié, relégué dans ma cour?»

22. La rhétorique et la prudence se dissocient alors; voir sur ce point M. Fumaroli dans "Aujourd'hui : facettes d'une renaissance de la rhétorique », Histoire de la rhétorique dans l'Europe moderne, Paris, PUF, 1999, p. 1284-1285 : «Les affaires humaines, selon Perelman, ne pourraient rentrer dans la sphère du raisonnable, la phronèsis des Grecs, la prudentia des Latins, que si l'on admettait la légitimité philosophique d'un art d'argumenter moins tranchant et péremptoire que la logique formelle : un art d'argumenter opératoire dans l'ordre fuyant, toujours particulier et imprévisible, qui règne dans la vie politique et dans la vie privée et qui est propre à établir, non pas la vérité absolue étrangère aux réalités humaines, mais du moins des évaluations argumentées et crédibles, des hypothèses vraisemblables appropriées à la prudence. »

23. III, 1 : «J'irai, n'en doutez point, le montrer à l'armée, / Plaindre aux yeux des soldats son enfance opprimée, / Leur faire, à mon exemple, expier leur erreur. / On verra d'un côté le fils d'un empereur / Redemandant la foi jurée à sa famille, / Et de Germanicus on entendra la 
fille ; / De l'autre, l'on verra le fils d'Ænobarbus, / Appuyé de Sénèque et du tribun Burrhus, / Qui tous deux, de l'exil rappelés par moi-même, / Partagent à mes yeux l'autorité suprême. / De nos crimes communs je veux qu'on soit instruit; / On saura les chemins par où je l'ai conduit. / Pour rendre sa puissance et la vôtre odieuses, /J'avouerai les rumeurs les plus injurieuses:/Je confesserai tout, exils, assassinats, / Poison même... »

24. Rhétorique, op. cit., II, I, $1377 \mathrm{~b} 19$.

25. V.969; voir également ce terme dans La Thébaïde, opposé par Polynice à Antigone, en II, 3, v. 519-523 : «N'imputez point ce crime à mon âme affligée ; / Dites plutôt, ma sœur, que vous êtes changée, / Dites que de mon rang l'injuste usurpateur / M'a su ravir encor l'amitié de ma sœur. / Je vous connais toujours et suis toujours le même. »

26. La première scène de l'acte III est d'abord occupée par le discours fleuve du roi à ses fils, exposant et justifiant son projet de marcher sur Rome. Après une concession admirative devant " cette grande entreprise », Pharnace met en doute la pertinence et la solidité d'un tel projet avant de repousser son corollaire, le projet du mariage avec l'héritière parthe et de proposer de choisir le pacte avec Rome. C'est là le pivot de la scène puisqu'une telle proposition provoque l'indignation de Xipharès qui instrumentalise le détour de son frère, et donc redouble en fait ce détour, à son profit, en se présentant comme le fils inconditionnellement fidèle à son père (v. 906-911) : «Rome, mon frère! O ciel! qu'osez-vous proposer?/Vous voulez que le roi s'abaisse et s'humilie ? / [...] Continuez, Seigneur. Tout vaincu que vous êtes [...]. » En demandant à prendre la tête de l'expédition, Xipharès accomplit, plus que la volonté de son père, celle de Monime mais, pour l'heure, son père ne l'« entend" pas encore et ne défait pas encore son discours.

27. «La ruse oratoire dans les tragédies de Racine », Cahiers de littérature du XVII ${ }^{e}$ siècle, 6, 1984, p. 115-123.

28. Ibid., p. 51.

29. "Rhétorique et tragédie au XVII ${ }^{\mathrm{e}}$ siècle ", XVII Siècle, 80-81, 1968, p. 89-105. Jacques Morel distingue au théâtre "une rhétorique explicite puisque consciente d'elle-même et seconde puisque ne s'adressant au spectateur que par la médiation d'un interlocuteur »: lorsque "le dialogue se présente [...] comme un combat où les personnages entendent se persuader mutuellement. Ils sont alors censés utiliser en toute connaissance de cause les moyens propres à convaincre un adversaire"; et d'autre part une rhétorique "implicite, puisque le héros sera censé s'exprimer "naturellement", et primaire, puisqu'elle ne pourra être attribuée qu'au talent du dramaturge et s'adressera directement au spectateur » quand « le poète entendra persuader son auditeur en faisant agir et parler devant lui un personnage ayant certaines passions et certains intérêts ». Il envisage la superposition de ces deux formes de la rhétorique "lorsque derrière les moyens de convaincre visiblement utilisés par un personnage le spectateur sera invité à découvrir l'expression de ses passions ou de ses intentions secrètes. »

30. Britannicus, II, 6, v. 639 sq. : "Cette sincérité sans doute est peu discrète, / Mais toujours de mon cœur ma bouche est l'interprète. / Absente de la cour je n'ai pas dû penser, / Seigneur, qu'en l'art de feindre il fallut m'exercer. [...]»; Néron persiste: «Il vaut mieux que luimême / Entende son arrêt de la bouche qu'il aime. / Si ses jours vous sont chers, éloignez-le de vous / Sans qu'il ait aucun lieu de me croire jaloux. »

31. V.1344-1349. Ce faisant, Monime met en perspective de manière très ironique le jugement auquel elle appelle alors Mithridate : «Et je vois quels malheurs j'assemble sur ma tête. / Mais le dessein est pris, rien ne peut m'ébranler. /Jugez-en, puisque ainsi je vous ose parler, / [...] / Après cela, jugez. Perdez une rebelle [...]» (v. 1360-1371).

32. Voir Burrhus face à Agrippine en Britannicus, I, 2, v. 279-282 : « Madame, je vois bien qu'il est temps de me taire, / Et que ma liberté commence à vous déplaire. / La douleur est injuste, et toutes les raisons / Qui ne la flattent point aigrissent ses soupçons. »

33. Voir J. Scherer, La Dramaturgie classique en France, Paris, Nizet, 2001, p. 245 sq. 
34. II, 5, v. 625-630; à comparer avec la scène IV, 4 de Britannicus où Narcisse pousse Néron à externaliser le processus de délibération, et donc la décision, en jouant précisément sur la conquête d'une autonomie délibérative par l'empereur figuré en orateur maître de son discours comme de son auditoire: "Mais Narcisse, dis-moi, que veux-tu que je fasse?» "Mais de tout l'univers quel sera le langage?» — «Et prenez-vous, Seigneur, leurs caprices pour guides? » « De vos propres désirs perdrez-vous la mémoire ? / Et serez-vous le seul que vous n'oserez croire?» «Ou plutôt ils n'ont tous qu'une même pensée:/Ils verraient par ce coup leur puissance abaissée;/Vous seriez libre alors, Seigneur; et devant vous,/Ces maîtres orgueilleux fléchiraient comme nous. / Quoi donc ? ignorez-vous tout ce qu'ils osent dire ? « Ah ! ne voulezvous pas les forcer à se taire?»

35. C'est le rôle du topos déployé par Antigone contre Créon en V, 3, v. 1285-1292 : «N'imputez qu'à vous seul la mort du roi mon frère, / Et n'en accusez point la céleste colère. / À ce combat fatal vous seul l'avez conduit: / Il a cru vos conseils, sa mort en est le fruit. / Ainsi de leurs flatteurs les rois sont les victimes; /Vous avancez leur perte en approuvant leurs crimes ; / De la chute des rois vous êtes les auteurs ; / Mais les rois en tombant entraînent leurs flatteurs. »

36. Cicéron, De l'orateur, II, 28, pour définir la rhétorique : «Sachez donc qu'il s'agit ici d'un art qui repose sur le mensonge, qui parvient rarement à la connaissance du vrai, qui cherche à exploiter les opinions et souvent même les erreurs des hommes. »

\section{AUTEUR}

\section{DELPHINE REGUIG}

Université Paris Sorbonne - Paris IV / CELLF $17^{\mathrm{e}}-18^{\mathrm{e}}$ 